\title{
Finite volume solution for two-phase flow in a straight capillary
}

\author{
Alexander Yelkhovsky and W. Val Pinczewski ${ }^{1}$ \\ ${ }^{1}$ School of Petroleum Engineering, University of New South Wales, Sydney 2052, Australia
}

\begin{abstract}
The problem of two-phase flow in straight capillaries of polygonal cross section displays many of the dynamic characteristics of rapid interfacial motions associated with pore-scale displacements in porous media. Fluid inertia is known to be important in these displacements but is usually ignored in network models commonly used to predict macroscopic flow properties. This study presents a numerical model for two-phase flow which describes the spatial and temporal evolution of the interface between the fluids. The model is based on an averaged Navier-Stokes equation and is shown to be successful in predicting the complex dynamics of both capillary rise in round capillaries and imbibition along the corners of polygonal capillaries. The model can form the basis for more realistic network models which capture the effect of capillary, viscous and inertial forces on pore-scale interfacial dynamics and consequent macroscopic flow properties.
\end{abstract}

PACS numbers: 47.56.+r,47.61.Jd

\section{INTRODUCTION}

The computational efficiency of pore network models has seen them increasingly used to estimate important macroscopic multiphase flow properties for porous media. The models are based on a simplified representation of the complex pore space and simplified physics to describe the pore-scale configuration and motion of the fluids in the pore space. Extensive reviews of the more recent developments in network modeling can be found in Refs. [1-5].

The more sophisticated network models represent the pore space as a network of interconnected angular flow channels which retain some of the more important geometrical and topological features of the actual pore space and allow the presence of partially saturated pores where wetting fluid is retained in corner films. The swelling of these films is important in imbibition where it results in snap-off and the creation of residual nonwetting fluid [68]. The movement of fluids in the network is assumed to occur in a quasisteady sequence of equilibrium states governed by capillary pressure (quasistatic models) or by a combination of capillary and viscous forces (dynamic models). In dynamic network models the effect of viscosity is modeled as a perturbation in capillary pressure determined from a pressure solution for steady-state flow in the network.

A growing body of literature now exists questioning the validity of the assumptions that the temporal evolution of fluid configurations can be modeled by a sequence of equilibrium states and that the pore-scale fluid motions leading to these states are steady. Recent studies of porescale displacements in two-dimensional micromodels using high-speed photography [9-11] and in sandstone samples using fast x-ray computed micro-tomography [12-16] show that the flow is inherently unsteady and that rapid interfacial motions associated with pore-scale displacement events influence fluid topology and thus macroscopic flow properties. Well-known examples of those events are the wetting film snap-off induced by the film swelling during imbibition, and the spontaneous displacement of wetting fluid from a pore by nonwetting fluid from an adjoining pore throat (Haines jump [17]).

Moebius and Or [9, 11] show that in micromodel displacements Haines jumps are associated with interfacial oscillations, fluid rearrangements, and simultaneous cascade-like displacement events in pores in the vicinity of the initial Haines jump. They attribute this behavior to the role of local inertial forces in pore-scale displacements [11]. Armstrong and Berg [10] report similar observations in their micromodel experiments. They show that during the initial phase of a jump the interface is rapidly accelerated to a peak velocity approximately three orders of magnitude greater than the average displacement velocity. Moreover, they find no correlation between the interfacial velocities and the mean displacement velocity. They also conclude that the displacements are dominated by fluid inertia at the pore scale. Similar observations are reported by Berg et al. [12] for displacements in actual sandstones using high-speed x-ray computed microtomography. They show that Haines jumps typically cascade through 10-20 pores and conclude that the motion at the pore scale is capillary-inertial controlled with time scales of the order of a millisecond consistent with acoustic measurements reported by DiCarlo et al. [18]. Rücker et al. [14] describe similar rapid interfacial motions for imbibition displacements in sandstone. They observed local snap-off events that caused meniscus oscillations and fluid rearrangements in distant pores within the same cluster and concluded that inertial forces are also important in imbibition.

The visualization studies are supported by computational studies on single pores and small two-dimensional networks. Ferrari and Lunati [19] used the Volume of Fluid method to numerically solve the Navier-Stokes equations and track the evolution of the interface in the corner of an angular pore. Their computations showed that local velocities can be orders of magnitude greater than the injection velocity and that they induce damped oscillations of the interface which depend only on fluid properties and pore geometry. The oscillations were of 
sufficient amplitude and duration to affect the order of invasion and the macroscopic distribution of fluids in the network. Armstrong et al. [20] used the density functional hydrodynamics method to simulate their micromodel observations. The computed results were in agreement with their experimental observations, and they conclude that temporal resolutions fine enough to resolve millisecond events are required to fully capture the effects of the unsteady flow on macroscopic flow properties.

The above studies clearly show that the flow of fluids in a network of interconnected pores is inherently unsteady and that inertial effects during rapid pore-scale displacements are important in determining the final configuration of fluids in both drainage and imbibition. Existing dynamic network models do not capture the unsteadiness of the flow and neglect important inertial effects associated with rapid pore-scale fluid reconfigurations. Meakin and Tartakovsky [21] suggest that these rapid fluid reconfigurations also invalidate the capillary dominated quasistatic assumption since dynamic (inertial) effects are also present in the limit of slow flow.

The importance of inertial effects for the capillary rise phenomenon was previously emphasized by Quéré et al. [22]. We agree with the authors of Ref. [22] that some experimental results cannot be explained without an account of inertia. However, we disagree with the theoretical approach of Ref. [22], which includes the energy conservation law applied to an open system, dissipation of flow energy without viscosity, and solution-dependent equations of motion.

The present study describes a numerical model for the spatial and temporal evolution of the interfaces in a capillary occupied by two immiscible fluids. The model can form the basis of a dynamic network model which includes capillary, viscous, and inertial forces. This is demonstrated by applying the model to the problems of capillary rise in a round tube whose end is brought into contact with the surface of a liquid, and the imbibition along the corners of a polygonal capillary induced by switching off the gravitational field. Quéré [23] reports experimental measurements of meniscus position as a function of time in a round tube for three liquids of different viscosities. The data display many of the characteristics of the dynamic pore-scale displacements discussed above - the importance of inertial forces and the occurrence of damped oscillations if the liquid viscosity is low enough. For the second problem the model simulations are compared with data reported by Weislogel and Lichter [24] for imbibition in cells of polygonal cross section and the experiments reported by Dong and Chatzis [25] for the flow of a wetting liquid along the corners of a square capillary. For clarity the model and solution method are described for the case of a zero contact angle. The general case of a dynamic contact angle is considered when the simulation results are compared with the measured data.

A sketch of the liquid phase configuration is shown at Fig. 1 for a tube with square cross section and rounded corners. Below we discuss behavior of the liquid-gas interface in terms of its elements, the meniscus (a rounded cap at the bottom of the interface), and the surface of corner films protruding above the meniscus. For a round tube there are no corner films, and the interface consists of only the meniscus.

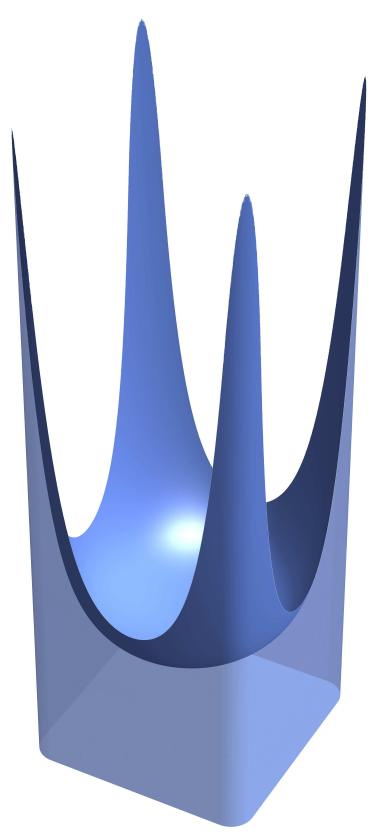

FIG. 1. Sketch of the liquid phase configuration.

\section{METHOD}

To mimic a pore network, we represent the tube as a chain of fixed size flow channels. Assuming that the tube radius determines a characteristic scale of the flow, we choose the length of each channel to be of the order of the tube radius.

Although this choice of discretization (as well as the choice of the variables below) might be suboptimal for the particular problem of capillary flow in a tube of constant cross section, it is motivated by the prospect of rather straightforward generalization of the method to proper networks.

In this section we outline our method according to the following sequence. We start with a minimal set of variables describing the interface position at any moment in time. Since the interface motion is governed by local fluxes, we derive the corresponding equations of motion clearly stating the approximations made on the way. Our treatment of pressure differs from the approaches commonly used in network models, and we devote a special subsection to explain the procedure. We continue with descriptions of interface transitions between flow channels and of the contact angle dynamics. Finally, we conclude with brief notes on our numerical scheme. 


\section{A. Interface}

The fluids are assumed to be incompressible. The evolution of the fluid-fluid interface is governed by the volume balance equation,

$$
\frac{d V}{d t}+\sum f=0
$$

where $V$ is a subvolume of either of two phases, and $\sum f$ is total outflux of the same phase through the subvolume boundaries.

We parametrize the interface geometry with a discrete set of variables consisting of the following subsets.

Along the tube, bulk liquid is separated from bulk gas by the meniscus. We define the meniscus position by an intersection of the spherical cap with the tube inner surface. Meniscus position along the tube comprises the first subset of interface variables. For a round tube, it is also the only one.

Behind the meniscus in an angular tube, we have gas in the bulk and liquid film in the corners. If the film spreads over several flow channels, its curvature radii at the channels joints comprise the second subset of interface variables. Below we call these film hosting joints the anchors.

Finally, in a tube with rounded corners (like the one shown in Fig. 1), a film can spread only up to the point where its curvature reaches that of the corner, $1 / r_{\text {corner }}$. This point is called film tip, and its position comprises the third subset of interface variables.

Figure 2 shows part of the longitudinal section of the flow. This section cuts along the axis (dashed line at the figure) and a corner of the tube (shown as the thick black line). The part of the tube shown in Fig. 2 consists of three flow channels of the same length; the left channel hosts the meniscus, the right one hosts the tip, and the film in between is anchored by two joints.

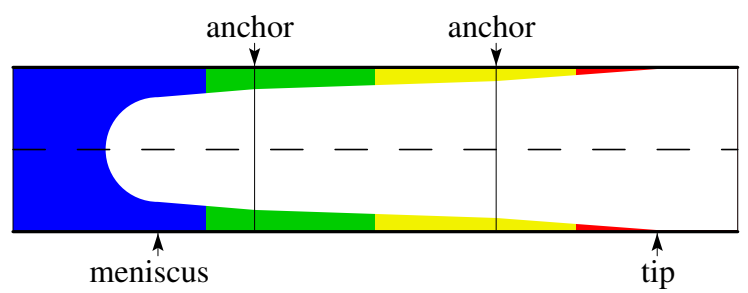

FIG. 2. Longitudinal section of the flow. Arrows point to discretization nodes. Colors indicate the associated subvolumes.

To associate one balance equation (1) with each variable, we need one subvolume per a variable. For an anchor, we draw cuts bounding its subvolume across the films midway between the anchor and each of its closest neighbors (either another anchor or the meniscus or the tip). Then for an anchor or a tip, $V$ in Eq. (1) is the liquid volume between the corresponding cuts, while $\sum f$ is a sum of liquid outfluxes through those cuts. On
Fig. 2, the longitudinal section of the anchor subvolumes are shown in green and yellow, while the meniscus and the tip subvolumes in blue and red, respectively.

For the meniscus, $V$ is a volume of liquid between the meniscus and the cut midway to the neighboring anchor, plus the part of the channel volume in front of the meniscus. We have to include the latter because it changes when the meniscus moves. The total outflux for the meniscus is the liquid outflux through the cut plus the liquid flux in front of and directed away from the meniscus. By virtue of volume conservation, the latter equals the total flux and does not depend on the cross section position.

When a flow starts, subvolumes associated with the interface variables start to change, so that the meniscus and the tip start to move along the tube, while the corner films start to swell. At some moment, the meniscus or the tip can cross a boundary of a hosting channel leading to a change in a number of anchors. In a round tube, the meniscus just moves from one channel to another.

If subvolumes are chosen in the manner described above, we obtain a well-defined system of coupled equations for the interface variables. Volume balance is provided by the fact that for each cut an outflux for a subvolume on one side is an influx for a subvolume on the other.

Once all subvolumes are known from the system of Eqs. (1), we update values of all interface variables (meniscus and tip positions and anchor radii) making up those subvolumes. For a round tube, there is a trivial relation between the meniscus position and a volume of liquid in front of it. For an angular one, we also need to choose a smooth interpolation of the film curvature between neighboring anchor points. The linear behavior of capillary pressure is the most natural choice for this.

\section{B. Fluxes}

To close the system of balance equations (1) we need to know the liquid flux through each of the cuts as well as the total flux through the tube.

To calculate the fluxes, we start with the Navier-Stokes equation for an incompressible fluid:

$$
\frac{\partial \boldsymbol{v}}{\partial t}+(\boldsymbol{v} \nabla) \boldsymbol{v}-\nu \Delta \boldsymbol{v}+\frac{\boldsymbol{\nabla} P}{\rho}-\boldsymbol{g}=\mathbf{0}
$$

Here $\boldsymbol{v}$ is the fluid velocity, $P$ is the fluid pressure, $\boldsymbol{g}$ is an apparent acceleration due to a body force (e.g., gravity), while $\rho$ and $\nu$ are the fluid density and kinematic viscosity, respectively. Here and below two vectors next to each other form the scalar product; the Laplacian is defined in the standard way, $\Delta \equiv \nabla^{2}$.

By definition, a fluid flux $f$ through a surface $\boldsymbol{a}$ is the integral $\int \mathrm{d}^{2} \boldsymbol{a} \boldsymbol{v}$ of fluid velocity $\boldsymbol{v}$ relative to this surface. The Einstein equivalence principle allows us to account for the surface motion by substituting $\boldsymbol{g} \rightarrow \boldsymbol{g}-d \boldsymbol{u} / d t$, where $\boldsymbol{u}$ is the surface velocity. 
Integrating each term from the Navier-Stokes equation (2) over a cut surface transverse to the channel axis, we get

$$
\dot{f}+2 \int \mathrm{d} a v v^{\prime}-\nu \int \mathrm{d} a \Delta v+\left(\frac{P^{\prime}}{\rho}-g+\dot{u}\right) a=0,
$$

where $v$ is the fluid velocity component along the axis direction $\boldsymbol{n}, g=\boldsymbol{g} \boldsymbol{n}$ and $u=\boldsymbol{u n}, a=\int \mathrm{d} a \equiv \int \mathrm{d}^{2} \boldsymbol{a} \boldsymbol{n}$ is the surface area, the dot and the prime denote partial derivatives over time and over the axial coordinate, respectively.

If one phase fills a tube cross section completely, parameter $a$ in Eq. (3) is the area of this cross section, $a_{\text {tube }}$. It is convenient to introduce the dimensionless parameter $\Pi$ characterizing the shape of the cross section, $\Pi=a_{\text {tube }} / R^{2}$, where $R$ is the radius of the largest sphere fitting into the tube.

Then, if the liquid forms corner films, their cross sectional area $a_{\text {film }}$ is a function of the film curvature radius $r$. For sharp corners, a dimensional consideration gives $a_{\mathrm{film}}(r)=\Pi_{\mathrm{film}} r^{2}$, where $\Pi_{\mathrm{film}}$ is a function of the corner angle. For rounded corners, we subtract the area of the sharp tip (see Fig. 3):

$$
a_{\text {film }}(r)=\Pi_{\text {film }}\left(r^{2}-r_{\text {corner }}^{2}\right),
$$

so that $\Pi_{\text {film }}=(\Pi-\pi) /\left(1-r_{\text {corner }}^{2} / R^{2}\right)$ according to the condition $a_{\text {tube }}=\pi R^{2}+a_{\text {film }}(R)$.

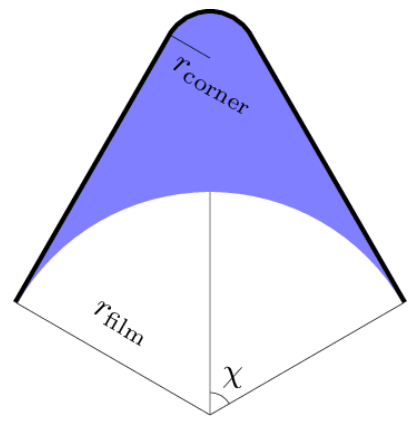

FIG. 3. Geometry of the film flow cross section.

We would like to write an approximation for Eq. (3) in terms of flux and cross section geometry only.

First, the convective acceleration term $2 \int \mathrm{d} a v v^{\prime}$ can be safely neglected for the slow film flow. For the bulk flow, we approximate it as $k f(f / a)^{\prime}$ where $k$ is 2 for the flat velocity profile and $8 / 3$ for the hyperbolic (Poiseuille) one.

Then, for the velocity $v$ we use the standard approximation of the steady-state flow profile, i.e., a solution of the 2D Poisson equation $\Delta_{\perp} v=$ const, where $\Delta_{\perp}$ is the Laplacian in two dimensions perpendicular to the flow. The transverse viscosity term $-\nu \int \mathrm{d} a \Delta_{\perp} v$ can then be rewritten as $\nu \beta f / s^{2}$, where $s$ is a characteristic size of the flow cross section (radius of the tube for the bulk, film curvature radius for the corner flow), and the flow resistance factor $\beta$ is a dimensionless function of the cross section geometry encoding information about the steady-state solution [26]. This factor can be calculated as $a / \int \mathrm{d} a w$, where $w$ is the solution to the equation $\Delta_{\perp} w=-1 / s^{2}$ with nonslip boundary condition at the wall and the full slip condition at the gas-liquid interface.

For two particular cross section geometries of angular tubes discussed in this paper, we could interpolate $\beta$ between values calculated at several points of $\zeta=$ $r_{\text {corner }} / r_{\text {film }}$. In a general case of a network with channels of various geometries, it is more efficient to have an analytical approximation for $\beta$ as a function of both $\zeta$ and the cross section angle $\chi$ :

$$
\beta_{\text {appr }}=\frac{4.4}{c}\left(\frac{1}{h_{x}^{2}}+\frac{1}{h_{y}^{2}}\right),
$$

where $c=1-0.37(1-\zeta)^{2}(1+0.2 \sin (2 \chi)), \quad h_{y}=$ $(\sec (\chi)-1)(1-\zeta), h_{x}=\tan (\chi / 2)$ if $2 \zeta \cos ^{2}(\chi / 2)<1$ and $\sqrt{h_{y}\left(2 \zeta-h_{y}\right)}$ otherwise. Figure 4 shows the relative difference $1-\beta_{\text {appr }} / \beta_{\text {num }}$ between the approximate solution (5) and its numerical counterpart calculated with the help of the PLTMG package [27].

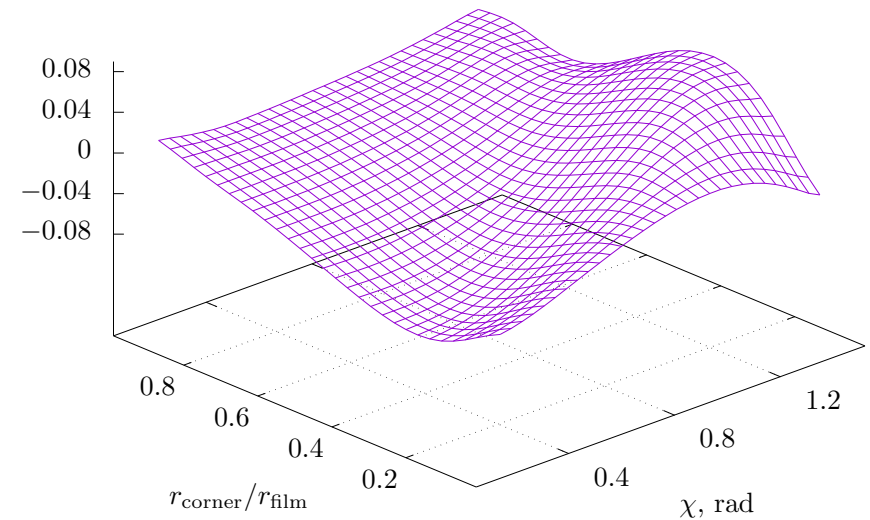

FIG. 4. $1-\beta_{\text {appr }} / \beta_{\text {num }}$ for the film flow resistance function $\beta$.

In contrast to the corner flow resistance factor which increases indefinitely for thin films, its counterparts for the bulk and single-phase flows both vary in finite ranges of the order of the round tube value $\beta=8$.

The last integral entering Eq. (3) we need to deal with is the longitudinal part of the viscous term, $-\nu \int \mathrm{d} a v^{\prime \prime}$. It vanishes for the single phase flow intervals since the fluids are incompressible and the channel cross section does not change along its axis. For the two-phase intervals, we use the incompressibility equation, $v^{\prime}+\nabla_{\perp} \boldsymbol{v}_{\perp}=0$, together with the Stokes theorem to rewrite this term as $\pm \nu \int \mathrm{d} b \dot{r}^{\prime}= \pm 2 \nu \Pi_{\text {film }} r \dot{r}^{\prime}$, where $r$ is the film curvature radius at the cross section. The integral is taken along the cross section boundary and the sign in front is plus for the corner flow and minus for the bulk flow.

Summing up, the approximate form of the Eq. (3) is

$$
\dot{f}+k f\left(\frac{f}{a}\right)^{\prime}+\nu \beta \frac{f}{s^{2}} \pm 2 \nu \Pi_{\text {film }} r \dot{r}^{\prime}+\left(\frac{P^{\prime}}{\rho}-g+\dot{u}\right) a=0 .
$$




\section{Pressure}

To close the system of Eqs. (6), we need to calculate pressure distributions along the flow. It is sufficient to find the pressure on the tube axis; pressure at the corners is determined by the condition $P_{\text {liq }}=P_{\text {gas }}-P_{\mathrm{c}}$, where $P_{\mathrm{c}}$ is a local capillary pressure of the film. Note that due to variation of the capillary pressure along the film, pressure gradients $P_{\text {liq }}^{\prime}$ and $P_{\text {gas }}^{\prime}$ can have opposite signs inducing counterflow of the two fluids.

Bulk pressure (that on the tube axis) can be calculated as follows. First, for a cross section located at a point $z$ along the tube we can apply Eq. (6) to the total flux $F$. For a single-phase cross section, $F$ is the flux of a phase at this cross section, for a two-phase one, $F=$ $f_{\text {liq }}+f_{\text {gas }}$. Separating the total flux time derivative and bulk pressure gradient terms from the rest and explicitly indicating $z$ dependence, we get:

$$
\dot{F}=-c(z) P_{\text {bulk }}^{\prime}(z)+\delta(z)
$$

If a section crosses a film, both $c$ and $\delta$ are the sums of bulk and corner contributions, otherwise they contain bulk contribution only (either of the liquid or of the gas).

Volume conservation requires that the total flux $F$ is the same for all cross sections of the tube. Hence, dividing Eq. (7) by $c(z)$ and integrating along the tube axis from the entrance to the exit, we get

$$
\dot{F} \int_{\text {entr }}^{\text {exit }} \mathrm{d} z \frac{1}{c(z)}=P_{\text {bulk }}^{\text {entr }}-P_{\text {bulk }}^{\text {exit }}+\int_{\text {entr }}^{\text {exit }} \mathrm{d} z \frac{\delta(z)}{c(z)}
$$

where $P_{\text {bulk }}^{\text {entr }}$ and $P_{\text {bulk }}^{\text {exit }}$ are the bulk pressure values at the tube entrance and at the exit, respectively. The pressure difference above is the sum of three terms: liquid pressure drop between the entrance and the meniscus, gas pressure drop between the meniscus and the tube exit, and the jump between the two which is equal to the meniscus capillary pressure.

For a tube touching the surface of a liquid bath we also need to take into account a flow of the liquid in the bath [28]. Assuming that this flow has only a radial component which does not depend on a direction inside the bath, we can integrate the radial component of the Navier-Stokes equation (2) along the radial coordinate to obtain

$$
\dot{F}+\frac{1}{2 a R} F^{2}-\frac{a}{\rho R} p-\frac{2 \nu}{3 R^{2}} F=0,
$$

where $\rho$ and $\nu$ are density and kinematic viscosity of the liquid, $R$ and $a$ are radius and area of a smallest hemisphere still allowing the above approximations for the flow, and $p$ is a pressure difference between infinity and this hemisphere. We assume that $R$ can be taken equal to the tube radius, while for $a$ we use the value $\pi R^{2}$ (instead of $2 \pi R^{2}$ ) and neglect a difference between $p$ and a pressure at the tube entrance.

Finally, to simulate flows at a constant applied pressure, we use Eqs. (8) and (9) with the constraint $p-$
$P_{\text {bulk }}^{\text {entr }}+P_{\text {bulk }}^{\text {exit }}=0$ to find the total flux rate throughout the evolution. To simulate flows at a constant flux rate, we use Eq. (8) to calculate the pressure drop between the tube ends. The pressure distribution along the tube can then be found from Eq. (7).

\section{Topology}

When the meniscus or the tip reaches a channel end, it disappears from that channel and reappears in the next one. If no new anchor appears as a result of this event, volume conservation is sufficient to find a new position of the meniscus or the tip. Otherwise, to determine a curvature radius at the new anchor we use the condition that the capillary pressure gradient is the same on both sides of the anchor.

\section{E. Contact angle}

Application of the above model to the capillary rise at the conditions of the experiments [23-25] quickly makes it apparent that an agreement with some of the measurements can only be achieved if we consider nonzero contact angles. Moreover, for the round tubes we also need to include the contact angle dynamics.

According to molecular kinetic theory, the correlation between contact angle $\theta_{\mathrm{d}}$, its value at rest $\theta_{\mathrm{s}}$, and the contact line velocity $w$ has the form (see Ref. [29] and references therein)

$$
\cos \theta_{\mathrm{d}}-\cos \theta_{\mathrm{s}}+\xi \mathrm{Ca}=0
$$

Here $\mathrm{Ca}=w \nu / \sigma$ is the contact line capillary number, $\nu$ is the liquid kinematic viscosity, and $\sigma$ is the liquidgas interface tension. The contact line friction factor $\xi$ cannot yet be calculated at the present state of the theory, and we use it as a fitting parameter to match our simulation results with experimental data.

An alternative correlation, based on the hydrodynamic analysis of the moving contact line problem [30, 31],

$$
\theta_{\mathrm{d}}^{3}-\theta_{\mathrm{s}}^{3}-\kappa \mathrm{Ca}=0
$$

where $\kappa$ is another friction factor to be used as a fitting parameter, gives slightly worse match of our results against experimental data, most probably because some of the flows have Reynolds number Re $\sim 10^{2}$, while the Voinov-Cox theory $[30,31]$ is applicable at $\operatorname{Re}<1$ only.

\section{F. Numerical scheme}

We compute the evolution of the interface using the fully implicit scheme with an adaptive time step. Each liquid subvolume is updated according to the balance equation (1). After extracting new values of the interface variables (including a new contact angle if necessary) we 
calculate a new pressure distribution as explained above. The liquid fluxes through the cuts between subvolumes are updated according to Eq. (6) (as well as the total flux if necessary). The iterations continue until new values of all variables are stable within tolerance limits.

To calculate the integrals from Eq. (8) we divide each flow channel into single- and two-phase intervals. For the flows considered in the present paper, each channel contains either two intervals if it hosts the meniscus or the tip, or just one interval otherwise. Then we approximate the integrals from Eq. (8) using the rectangle rule applied to those intervals, for example,

$$
\int_{\text {entr }}^{\text {exit }} \mathrm{d} z \frac{1}{c(z)} \approx \sum_{i} \frac{l_{i}}{a_{\text {bulk }, i} / \rho_{\text {bulk }}+a_{\text {film }, i} / \rho_{\text {film }}},
$$

where $l_{i}$ is interval length, while $a_{\mathrm{bulk}, i}=a_{\mathrm{tube}}-a_{\mathrm{film}, i}$ and $a_{\text {film }, i}$ are the areas of the flow cross sections through the middle of the interval, for the bulk and the film, respectively.

The simulations described below were carried out on a laptop with a Core 2 Duo processor and 8 Gb of memory. Most of the simulations take just a few second to run. The only exception is the Soltrol 100 imbibition process (see below), which took a few hours both to observe [25] and to simulate.

\section{RESULTS}

a. Round capillary We use measured heights of capillary rise for three liquids (viscous silicone oil, ethanol, and ether) from Ref. [23]. Comparisons between our computations and the data are shown in Figs. 5-7.

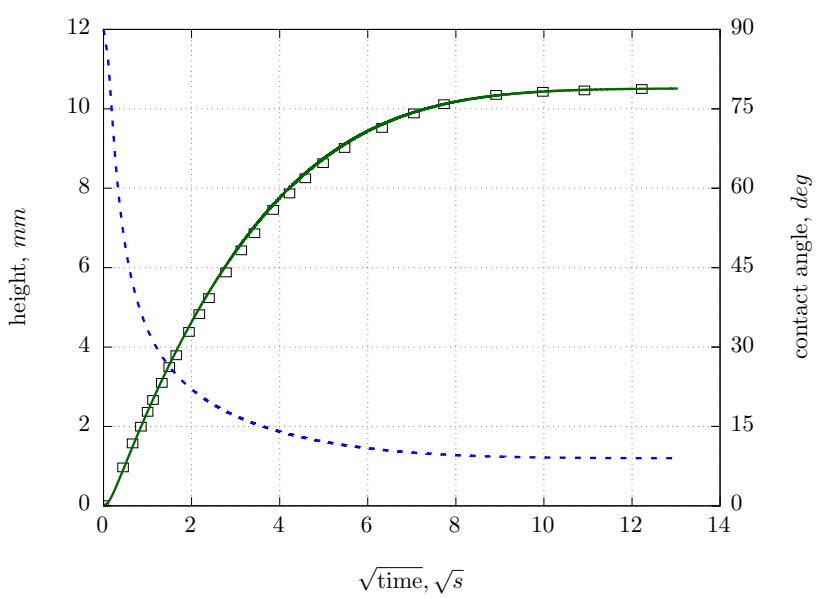

FIG. 5. Capillary rise of silicone oil (density $980 \mathrm{~kg} / \mathrm{m}^{3}$, viscosity $500 \mathrm{cP}$, air interface surface tension $21.1 \mathrm{mN} / \mathrm{m}$ ) in a glass tube of radius $421 \mu \mathrm{m}$. Rectangles are experimental data for the rise height from Ref. [23], and solid and dashed lines are simulation results for the height and dynamic contact angle, respectively.

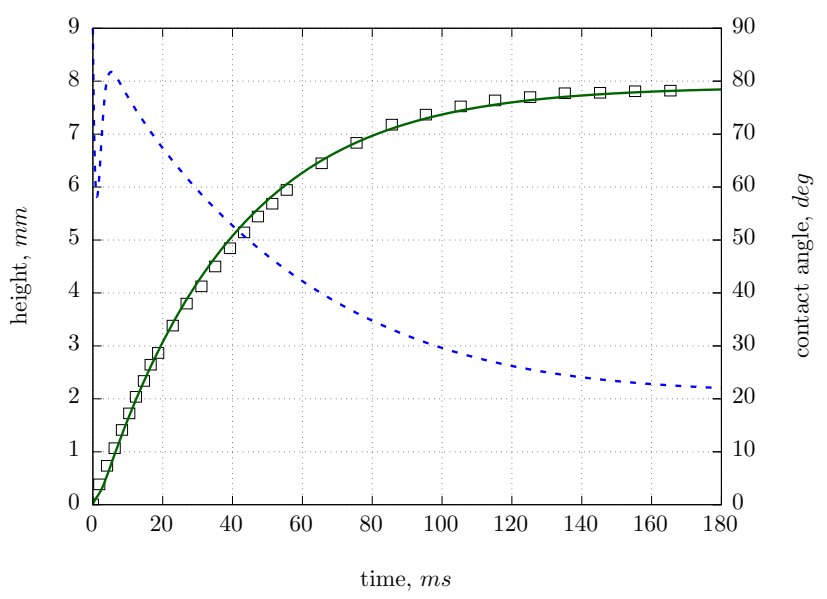

FIG. 6. Capillary rise of ethanol (density $780 \mathrm{~kg} / \mathrm{m}^{3}$, viscosity $1.17 \mathrm{cP}$, air interface surface tension $21.6 \mathrm{mN} / \mathrm{m}$ ) in a glass tube of radius $689 \mu \mathrm{m}$. Notations are the same as in Fig. 5 .

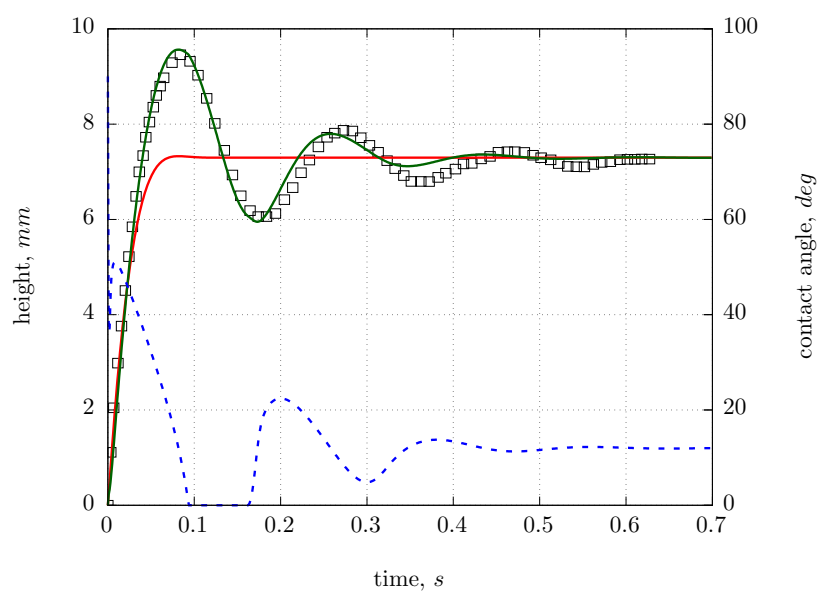

FIG. 7. Capillary rise of ether (density $710 \mathrm{~kg} / \mathrm{m}^{3}$, viscosity $0.3 \mathrm{cP}$, air interface surface tension $16.6 \mathrm{mN} / \mathrm{m}$ ) in a glass tube of radius $689 \mu \mathrm{m}$. Notations are the same as in Fig. 5 . Red line is calculated with the inertial term suppressed by $1 / 5$.

To match our results with experimental data we use the following procedure. First, the static contact angle $\theta_{\mathrm{s}}$ is extracted from the observed equilibrium height. Then, we run a few simulations with this $\theta_{\mathrm{s}}$ and various values of $\xi$ to get the initial slope of the capillary rise curve to match the observed one. We do not try to achieve the best possible match; our aim is to demonstrate that the model captures major physical features of the process.

It is not evident from Ref. [23] how the meniscus height is defined. The simulation results are presented for the height of the contact line above the surface of the liquid bath. We obtain the best agreement between our results and the measured data if we assume nonzero static contact angles $\theta_{\mathrm{s}}$, approximately $9^{\circ}$ for the silicone oil, $22^{\circ}$ for ethanol, and $12^{\circ}$ for ether. Also, we find that the best agreement with the measurements is achieved when 
the friction factor $\xi$ from (10) is approximately 5 for the silicone oil, 55 for ethanol, and 60 for ether.

Due to its low viscosity, the ether column overshoots its equilibrium height and then recedes. According to Ref. [32], behind the meniscus a receding column trails a film with thickness $3.72 \mathrm{Ca}^{2 / 3} R$, where $R$ is the tube radius. This film effectively increases the meniscus curvature.

To demonstrate importance of inertia, we ran a simulation with the flux time derivative term multiplied by $1 / 5$ (the convection term is zero because velocity profile is the same for all $z$ 's). The result shown in red at Fig. 7 clearly indicates that inertia is responsible for the height oscillations.

b. Polygonal capillary In a series of experiments described in Ref. [25], a 4 to $5 \mathrm{~cm}$ long slug of a wetting liquid was introduced from one end of the half-meter long tube with the square cross section. Then both ends of the tube were sealed, the tube was turned from vertical to horizontal position, and the motion of both the liquid-gas meniscus and the film tip was recorded.

We simulate the flow of Soltrol 100 through the tube with the transverse size of $500 \mu \mathrm{m}$ and rounded corners with radius of $51.2 \mu \mathrm{m}$. The best agreement with the experimental data is achieved with the contact angle $\theta_{\mathrm{s}}=2.5^{\circ}$. Figure 8 shows a comparison between the sim-

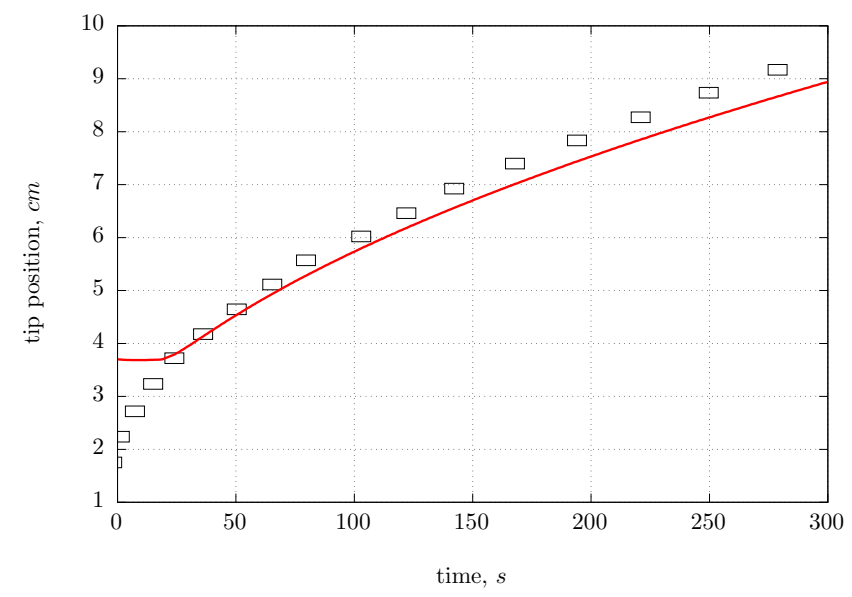

FIG. 8. Imbibition of Soltrol 100 (density $739 \mathrm{~kg} / \mathrm{m}^{3}$, viscosity $0.98 \mathrm{cP}$, air interface surface tension $22.0 \mathrm{mN} / \mathrm{m}$ ) along the corners of the $500 \mu \mathrm{m}$ square glass tube. Rectangles are experimental data for the tip displacement from Ref. [25]; solid line is our simulation result.

ulations and the measured data for the displacement of the film tip. We see a reasonable agreement after approximately $30 \mathrm{~s}$ from the start of the flow. Looking at the film profile for the earlier times, we see a swelling wave propagating from the meniscus and finally reaching the tip. Initial distance from the meniscus to the tip is denoted by $x_{0}$ at Ref. [25]. This distance can be calculated from the equation $\left(\rho_{\text {liq }}-\rho_{\text {gas }}\right) g x_{0}=\sigma / r_{\text {corner }}-\sigma / r_{\text {film }}$, where $r_{\text {film }}$ is the film radius at the meniscus. For Soltrol 100 we get $x_{0} \approx 3.7 \mathrm{~cm}$. It is not clear from Ref. [25] how exactly the tip position was measured to produce $x_{0} \approx 1.7 \mathrm{~cm}$. Moreover, Fig. 6 from Ref. [25] also shows a rather different value of $x_{0} \approx 1.2 \mathrm{~cm}$ for Soltrol 170 even though the relevant properties of two liquids are quite close: 0.739 versus $0.792 \mathrm{~g} / \mathrm{cm}^{3}$ for densities, and 22 versus 24 dyne/cm for surface tensions, respectively.

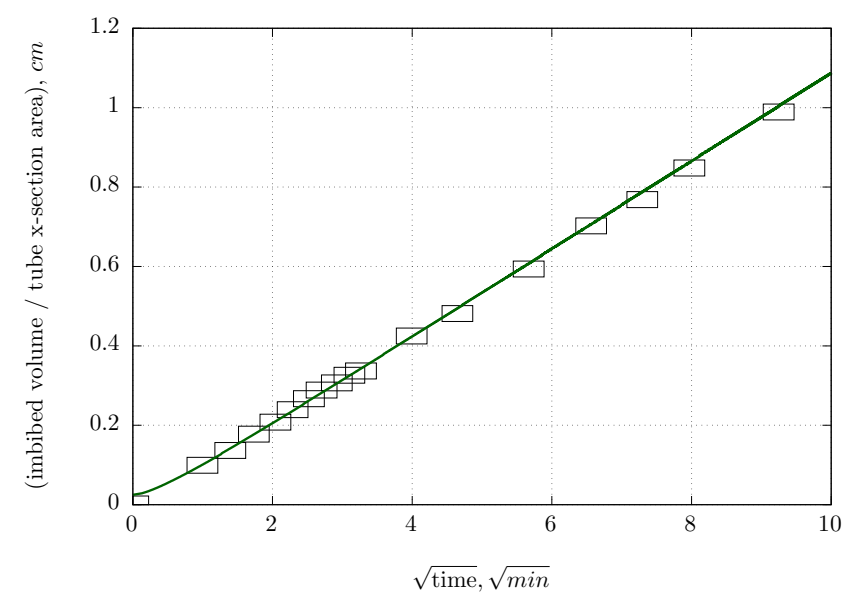

FIG. 9. Same flow as at Fig. 8, measurement data and simulation result for the absolute value of the meniscus apex displacement.

Figure 9 shows a comparison of our simulation result with the data for meniscus displacement.

In the experiments [24], acrylic test cells of polygonal cross section were partially filled with a silicone oil and then dropped from a $27 \mathrm{~m}$ high tower. Interface evolution during the 2.2 second free fall was filmed at 128 frames per second and then digitized.

We simulate the conditions for the ST10 experiment from Ref. [24] with $10 \mathrm{cS}(9.35 \mathrm{cP})$ silicone oil inside the $12 \mathrm{~mm}$ equilateral triangle tube. A comparison between the simulated and measured data is shown in Figs. 10 and 11 . The agreement between the two looks quite reasonable except for the meniscus apex position during the first approximately 0.16 seconds of zero-gravity conditions. According to Ref. [24], behavior of the system at the earlier times is governed by relaxation of the meniscus shape from almost flat initially (due to the gravity field) to that of a spherical cap (due to the surface tension). Since our primary interest is in small capillaries where gravity can be neglected, we do not account for the effect of gravity on meniscus shape.

Figure 12 from Ref. [24] (reproduced at Fig. 13) shows similar behavior observed in their experiment LT2. According to Ref. [24], the film profile behavior in general and an appearance of the constant height point in particular, are universal properties of the film flow along the tube corners.

Figure 12 shows how the film profile changes with time. In particular, after approximately 0.6 seconds from the start of the flow, all profiles come through the same point. The height of this point, $1.96 \mathrm{~mm}$, agrees well with the 


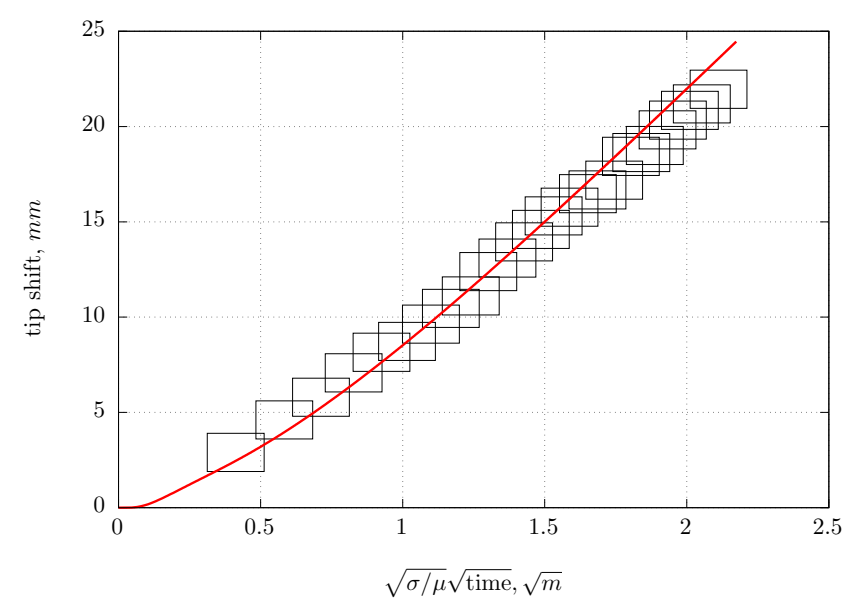

FIG. 10. Imbibition of silicone oil (density $935 \mathrm{~kg} / \mathrm{m}^{3}$, viscosity $9.35 \mathrm{cP}$, air interface surface tension $20.1 \mathrm{mN} / \mathrm{m}$ ) along the corners of triangle acrylic tube with $12 \mathrm{~mm}$ side. Rectangles are experimental data for the tip displacement from Ref. [24]; solid line is our simulation result.

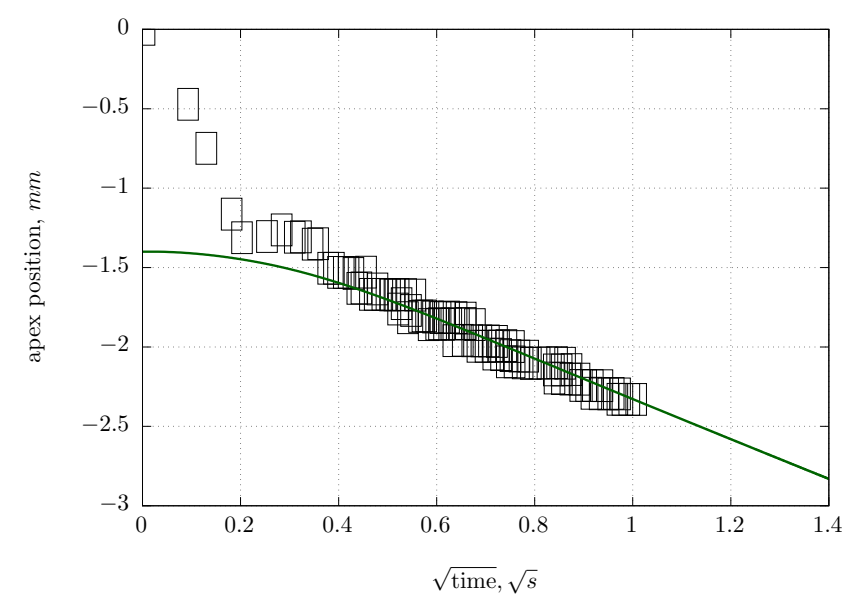

FIG. 11. Same flow as at Fig. 10, measurement data and simulation result for the meniscus apex position.

experimental value $1.97 \pm 0.08 \mathrm{~mm}$ [24] (see the insert in Fig. 12).

\section{CONCLUSIONS}

A simple one-dimensional numerical model, based on an averaged Navier-Stokes equation, is successful in predicting the dynamics of the flow in capillaries of circular and angular cross section. The inclusion of meniscus po- sition as a model parameter results in an efficient and accurate front-tracking scheme with sufficient temporal resolution to resolve the initial acceleration of the interface and subsequent inertia-driven oscillations. The mechanistic similarity between the onset of pore-scale interfacial displacements in porous media and capillary flow $[9,11]$ suggests that the numerical model can form

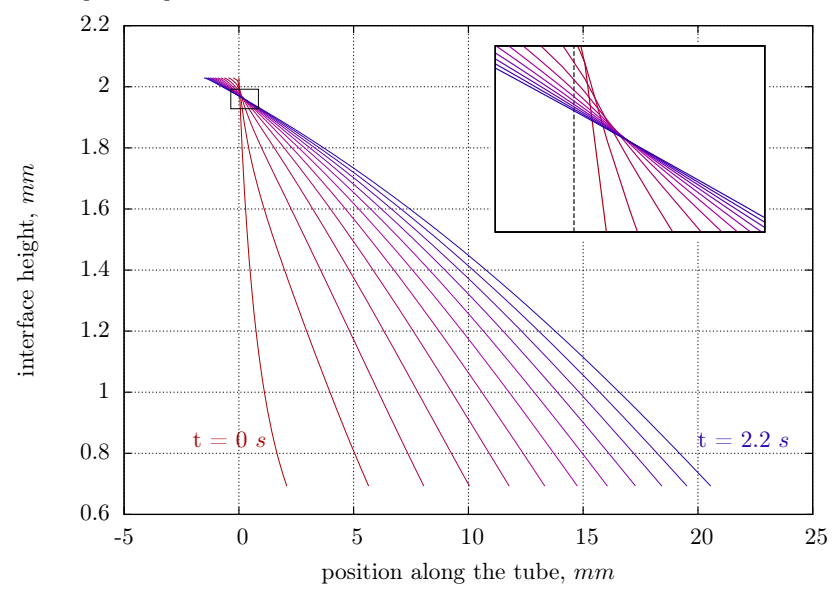

FIG. 12. Simulated profile of the interface height along the tube. Snapshots are made every 0.2 seconds. The insert shows a close-up of the constant height point.
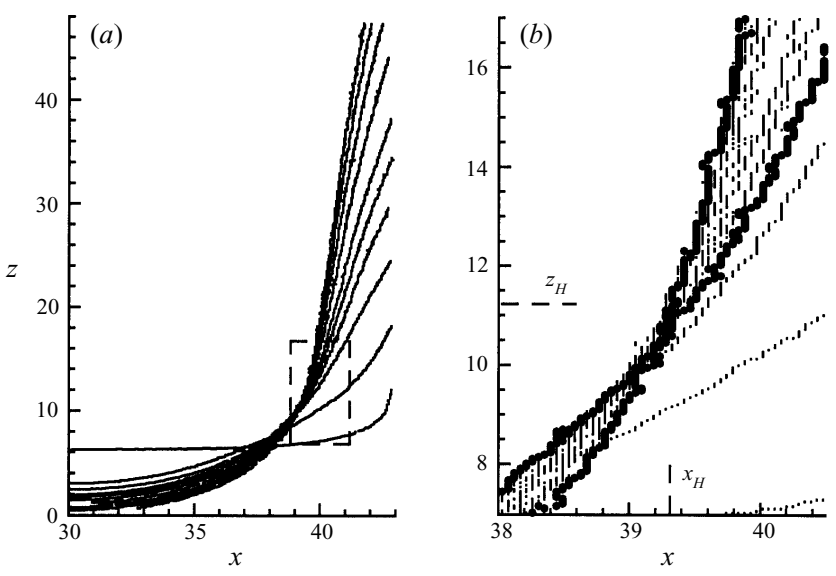

FIG. 13. Interface profiles measured in one of the experiments from Ref. [24]. Interface height is measured along $x$, and $z$ is position along the tube.

the basis for more realistic network models which capture the effects of capillary, viscous and inertial forces on pore-scale interfacial dynamics and consequent macroscopic flow properties.
[1] Martin J. Blunt, Branko Bijeljic, Hu Dong, Oussama Gharbi, Stefan Iglauer, Peyman Mostaghimi, Adriana Paluszny, and Christopher Pentland, "Pore-scale imag- ing and modelling," Adv. Water Resources 51, 197-216 (2013). 
[2] I. Bondino, G. Hamon, W. Kallel, and D. Kachuma, "Relative permeabilities from simulation in $3 \mathrm{~d}$ rock models and equivalent pore networks: Critical review and way forward," Petrophysics 54, 538-546 (2013).

[3] V. Joekar-Niasar and S. M. Hassanizadeh, "Analysis of fundamentals of two-phase flow in porous media using dynamic pore-network models: A review," Crit. Rev. in Env. Sci. Tech. 42, 1895-1976 (2012).

[4] Arash Aghaei and Mohammad Piri, "Direct pore-to-core up-scaling of displacement processes: Dynamic pore network modeling and experimentation," J. Hydrol. 522, 488-509 (2015).

[5] A.Q. Raeini, B. Bijeljic, and M.J. Blunt, "Generlaized network modelling: capillary dominated two-phase flow - model description," arXiv:1707.02788 [physics.compph].

[6] Viet Hoai Nguyen, Adrian P. Sheppard, Mark A. Knackstedt, and W. Val Pinczewski, "The effect of displacement rate on imbibition relative permeability and residual saturation," J. Petrol. Sci. Eng. 52, 54-70 (2006).

[7] Nasiru A. Idowu and Martin J. Blunt, "Pore-scale modelling of rate effects in waterflooding," Transp. Porous Media 83, 151-169 (2010).

[8] K. Singh, H. Menke, M. Andrew, Q. Lin, C.Rau M.J. Blunt, and B. Bijeljic, "Dynamics of snap-off and porefilling events during two-phase fluid flow in permeable media," Nature Sci. Rep. 7, 5192 (2017).

[9] Franziska Moebius and Dani Or, "Interfacial jumps and pressure bursts during fluid displacement in interacting irregular capillaries," J. Colloid Interface Sci. 377, 406415 (2012).

[10] Ryan T. Armstrong and Steffen Berg, "Interfacial velocities and capillary pressure gradients during haines jumps," Phys. Rev. E 88, 043010 (2013).

[11] Franziska Moebius and Dani Or, "Inertial forces affect fluid front displacement dynamics in a pore-throat network model," Phys. Rev. E 90, 023019 (2014).

[12] Steffen Berg, Holger Ott, Stephan A. Klapp, Alex Schwing, Rob Neiteler, Niels Brussee, Axel Makurat, Leon Leu, Frieder Enzmann, Jens-Oliver Schwarz, Michael Kersten, Sarah Irvine, and Marco Stampanoni, "Real-time 3d imaging of haines jumps in porous media flow," PNAS 110, 3755-3759 (2013).

[13] S. Berg, R.T. Armstrong, A. Georgiadis, H. Ott, A. Schwing, R. Neiteler, N. Brussee, A. Makurat, M. Rücker, L. Leu, M. Wolf, F. Khan, F. Enzmann, and M. Kersten, "Onset of oil mobilization and nonwettingphase cluster-size distribution," Petrophysics 56, 15-22 (2015).

[14] M. Rücker, S. Berg, R. T. Armstrong, A. Georgiadis, H. Ott, A. Schwing, R. Neiteler, N. Brussee, A. Makurat, L. Leu, M. Wolf, F. Khan, F. Enzmann, and M. Kersten, "From connected pathway flow to ganglion dynamics," Geophys. Res. Lett. 42, 3888-3894 (2015).
[15] Matthew Andrew, Hannah Menke, Martin J. Blunt, and Branko Bijeljic, "The imaging of dynamic multiphase fluid flow using synchrotron-based x-ray microtomography at reservoir conditions," Transp. Porous Media 110, 1-24 (2015).

[16] R.T. Armstrong, J.E. McClure, M.A. Berill, M. Rücker, S. Schluter, and S. Berg, "Flow regimes during immicible displacement," Petrophysics 58, 10-18 (2017).

[17] William B. Haines, "Studies in the physical properties of soil. v. the hysteresis effect in capillary properties, and the modes of moisture distribution associated therewith," The Journal of Agricultural Science 20, 97-116 (1930).

[18] David A. DiCarlo, Jose I. G. Cidoncha, and Craig Hickey, "Acoustic measurements of pore-scale displacements," Geophys. Res. Lett. 30, 1901 (2003).

[19] Andrea Ferrari and Ivan Lunati, "Inertial effects during irreversible meniscus reconfiguration in angular pores," Adv. Water Resources 74, 1-13 (2014).

[20] Ryan T. Armstrong, Nikolay Evseev, Dmitry Koroteev, and Steffen Berg, "Modeling the velocity field during haines jumps in porous media," Adv. Water Resources 77, 57-68 (2015).

[21] Paul Meakin and Alexandre M. Tartakovsky, "Modeling and simulation of pore-scale multiphase fluid flow and reactive transport in fractured and porous media," Rev. Geophys. 47, RG3002 (2009).

[22] David Quéré, Élie Raphaël, and Jean-Yves Ollitrault, "Rebounds in a capillary tube," Langmuir 15, 3679-3682 (1999).

[23] D. Quéré, "Inertial capillary," Europhys. Lett. 39, 533538 (1997).

[24] Mark M. Weislogel and Seth Lichter, "Capillary flow in an interior corner," J. Fluid Mech. 373, 349-378 (1998).

[25] M. Dong and I. Chatzis, "The imbibition and flow of a wetting liquid along the corners of a square capillary tube," J. Colloid Interface Sci. 172, 278-288 (1995).

[26] T.C Ransohoff and C.J Radke, "Laminar flow of a wetting liquid along the corners of a predominantly gasoccupied noncircular pore," J. Colloid Interface Sci. 121, 392-401 (1988).

[27] R. E. Bank, PLTMG: A software package for solving elliptic partial differential equations, users' guide 11.0, Tech. Rep. (University of California at San Diego, 2012).

[28] J Szekely, A.W Neumann, and Y.K Chuang, "The rate of capillary penetration and the applicability of the washburn equation," J. Colloid Interface Sci. 35, 273-278 (1971).

[29] Damien Duvivier, Terence D. Blake, and Joël De Coninck, "Toward a predictive theory of wetting dynamics," Langmuir 29, 10132-10140 (2013).

[30] O. V. Voinov, "Hydrodynamics of wetting," Fluid Dynamics 11, 714-721 (1976).

[31] R. G. Cox, "The dynamics of the spreading of liquids on a solid surface. Part 1. Viscous flow," Journal of Fluid Mechanics 168, 169-194 (1986).

[32] F. P. Bretherton, "The motion of long bubbles in tubes," J. Fluid Mech. 10, 166-188 (1961). 